\title{
Plasma Entry from the Flanks of the Near-Earth Magnetotail: GEOTAIL Observations in the Dawnside LLBL and the Plasma Sheet
}

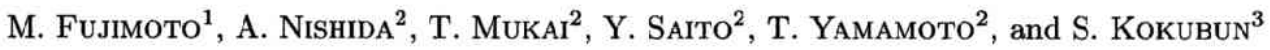 \\ ${ }^{1}$ Dept. Physics, Nagoya University, Nagoya 464-01, Japan \\ ${ }^{2}$ ISAS, Sagamihara, Kanagawa 229, Japan \\ ${ }^{3}$ STEL, Nagoya University, Toyokawa 442, Japan
}

(Received October 25, 1995; Revised February 23, 1996; Accepted March 1, 1996)

\begin{abstract}
GEOTAIL data from the low-latitude flanks of the magnetotail at $-30 R_{E}<X_{G S M}<$ $-15 R_{E}$ are analyzed. An example presented in this paper, which is representative of a group of the cases at the dawnside, shows that the region is characterized by highly varying magnetic field and the appearance of cold $(<1 \mathrm{keV})$ and dense $(>0.5 / \mathrm{cc})$ plasma. While some of these cold-dense plasma are detected to flow tailward at $300-500 \mathrm{~km} / \mathrm{s}$, others are found to flow only slowly tailward or even sunward sometimes. Electrons of $>100 \mathrm{eV}$ are seen to be depleted in the tailward flowing part, suggesting that these data are taken when the satellite was located on open field lines. When the sunward flows are detected, the denisty tends to show an intermediate value, and thermal electrons $(100-500 \mathrm{eV})$ are found to show bi-directional anisotropy. This suggests that part of the magnetosheath plasma is captured on closed field lines at the inner part of the low-latitude boundary layer (LLBL) in the tail flanks. Another study inside the plasma sheet at $\left(X_{G S M}, Y_{G S M}\right)=(-17,-12) R_{E}$ (a position well inside the magnetopause) shows a 2.5-hours period of the plasma sheet filled with cold $(500 \mathrm{eV})$ and dense $(2 / \mathrm{cc})$ plasma. Bi-directional thermal electrons are seen to accompany this population. This may imply that relatively unheated plasma from the LLBL of the near-Earth magnetotail is filling a substantial part of the plasma sheet.
\end{abstract}

\section{Introduction}

It is widely accepted that plasma from the ionosphere and that of the solar wind origin are the two major sources for the hot plasma trapped in the plasma sheet. As for the solar wind plasma, two entering processes have been considered: the entry from the high-latitude lobes and from the low-latitude flanks. In contrast to the vast knowledge on the high-latitude entry process, whose very origin goes back to the first paper on the open magnetosphere by Dungey (1961), considerably less is known about the low-latitude entry process. One of the reasons for this is the theoretical difficulty in understanding the plasma transport across the low-latitude magnetopause.

Observations in the dayside low-latitude boundary layer (LLBL) showing the mixture of magnetospheric and magnetosheath ions on closed field lines have been known for some time (e.g., Sckopke et al., 1981). However, the mechanism of this mixing, that is, the mixing that seems to take place without a change in the field line topology, is still under debate. Enhanced wave activity at the magnetopause current layer allowed Tsurutani and Thorne (1982) to propose that the mixing is brought about by diffusion due to wave-particle interactions, but the sharp gradients observed at the edge of the LLBL (e.g., Sckopke et al., 1981) do not fit into this picture. As for the observation in the magnetotail, leaving aside how the magnetosheath plasma penetrate to the closed field line region, Eastman et al. (1985) concluded that this boundary layer on closed field lines directly supplies low-energy ions to the central plasma sheet.

Less knowledge of the low-latitude entry process does not mean that the magnetotail flank has been treated as a minor contributor to the magnetotail plasma budget. Spence and Kivelson 
(1994) have developed a convection model of the magnetotail including the dawn flank as one of the plasma sources. In their model, the plasma sheet is assumed to be filled with plasma coming from the distant tail and from the dawnside flank (the entry process at the flank was unspecified). It was shown that the contribution from the flank can make the resultant plasma sheet much more closely resemble the observations, compared to the situation when only the distant tail source is taken into account.

The other reason for less knowledge about the LLBL has been the limitation of the data available. Williams et al. (1985) have shown that the LLBL in the tail consists of two layers, as in the dayside. Mitchell et al. (1987) and Traver et al. (1991) reported that the topology of the field lines in this region is controlled by the IMF $B_{z}$ component, closed (open) when northward (southward). All these tail-LLBL observations by the ISEE 1,2 satellites are limited to $X_{G S M}>-15 R_{E}$ so that studying the physics of the LLBL further down the tail with satellite data of an up-to-date performance was not possible. The Geotail satellite, owning to its welldesigned orbit, provides useful information on this region as it skims along the flanks of the magnetotail. Moreover, now that 3D particle data with much higher time resolution are available, we are able to uncover some processes that were previously unidentified.

In this paper, Geotail observations at the flank of the tail $-30 R_{E}<X_{G S M}<-15 R_{E}$ are reported. In accordance with previous studies but with a more complete data set, it is shown that low-latitude flanks of the tail is likely to be the site where a part of the magnetosheath plasma flowing past is captured on the closed field lines of the magnetosphere.

Having an evidence that the magnetotail flank is a possible source for the plasma sheet population, a question that comes up is how much the plasma from the flank contribute to the plasma budget of the magnetotail. In the latter half of this paper, we will show a dataset in the plasma sheet at $\left(X_{G S M}, Y_{G S M}\right)=(-17,-12) R_{E}$. The plasma sheet at this location is found to be filled with cold dense plasma, which is likely to be an unheated population coming from the flank. The least implication of this observation would be that the magnetotail flank should not be dismissed as a plasma source for the plasma sheet.

\section{LLBL Observations}

The data in Fig. 1 were obtained when GEOTAIL encountered with magnetosheath-like plasma around $\left(X_{G S M}, Y_{G S M}, Z_{G S M}\right)=(-27,-18,-3) R_{E}$. Shown are the three components of the magnetic field, the ion density and temperature, and the three components of the ion bulk flow. The spacecraft coordinates, which are essentially equivalent to the GSE coordinates, are used throughout the paper. The bottom three panels in Fig. 2 are the $E-t$ diagrams of sunward flowing ions, tailward flowing ions, and omnidirectional electrons, respectively. The ion denisty and the $V_{x}$ component of the ion flow are also shown for the sake of comparison. $12 \mathrm{sec}$. resolution data are used throughout this paper. Details of the instruments are given by Kokubun et al. (1994) and Mukai et al. (1994).

The plots start from the time when Geotail was inside the plasma sheet. Magnetosheathlike plasma, identified as high density and low temperature population in Fig. 1, are detected frequently in the interval 14:28-16:42 UT (between the vertical dashed lines in Fig. 1). This interval will be called "the boundary layer interval" hereafter and will be studied intensively in this section. The beginning of this interval is marked by the rise in the density (Fig. 1). The end of the interval is determined from the change in the electron anisotropy discussed later. GEOTAIL went back into the plasma sheet after this boundary layer interval. Not only from the GEOTAIL position but also from the fact that the interval of interest is sandwiched between the plasma sheet data, we infer that the boundary layer is located at the low-latitude part of the flank. The magnetic field was highly varying during the boundary layer interval. This is inconsistent with an alternative idea that the cold-dense plasma is the mantle population detected at the plasma 

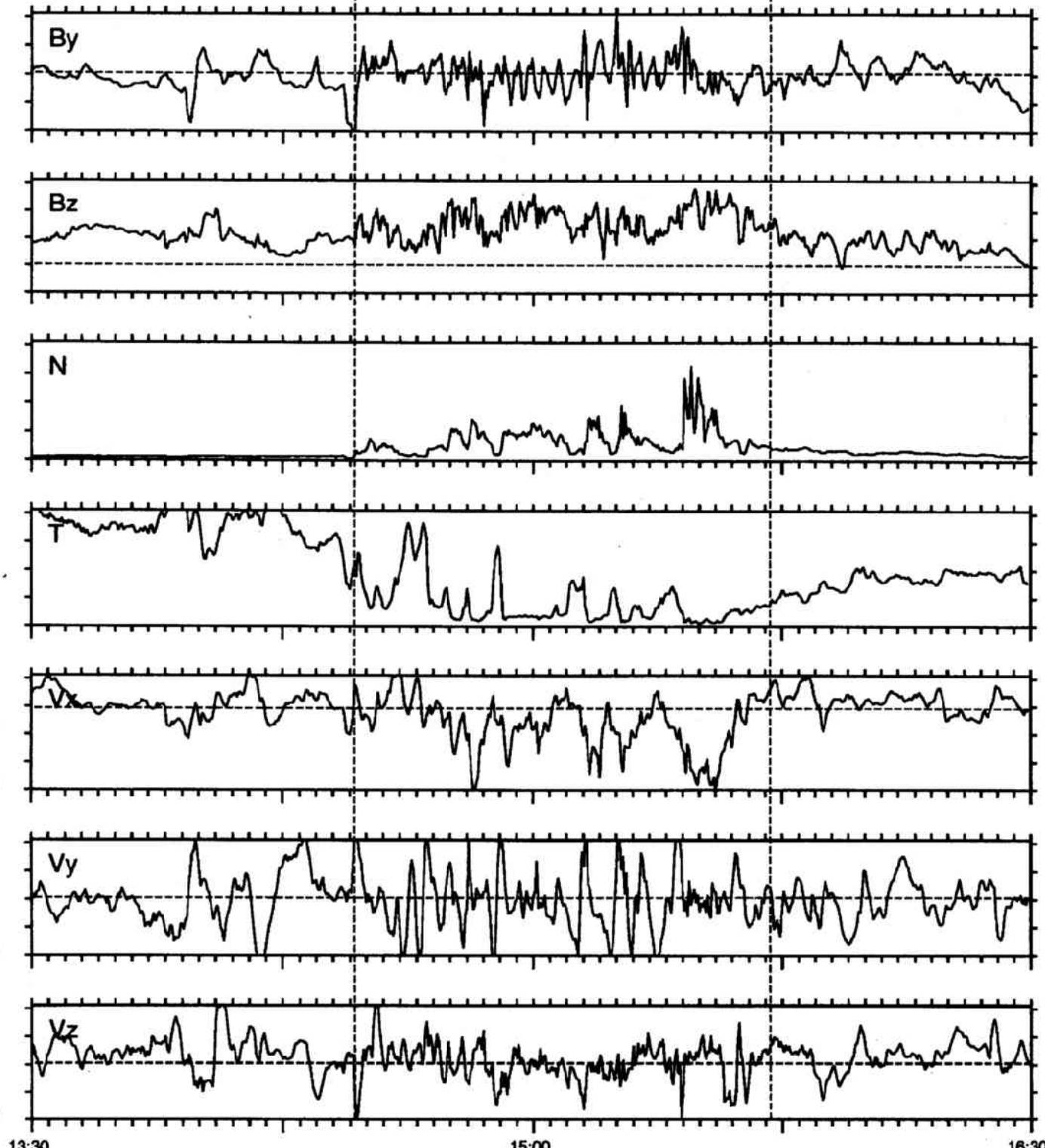

15:00

Fig. 1. An observation of dawnside LLBL at $X_{G S M} \sim-27 R_{E}$. From top, the three components of the magnetic field (nT), the ion density (/cc) and temperature (keV), and the three components of the ion bulk flow (km/s).

The boundary layer interval is between the two vertical dashed lines. 


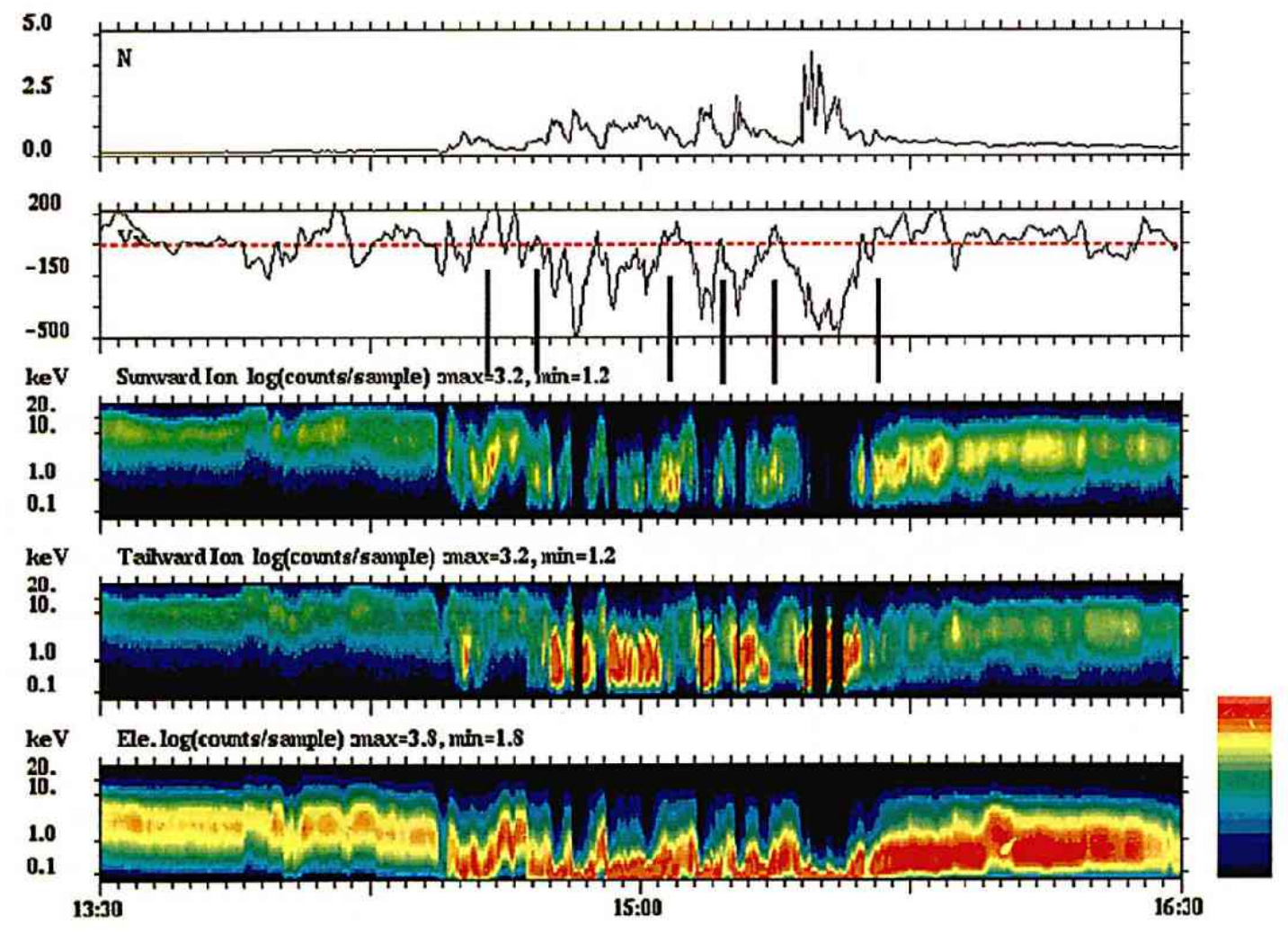

Fig. 2. E-t diagrams for sunward ions, tailward ions, and omnidirectional electrons, are shown with the panels for ion density $(/ \mathrm{cc})$ and the $V_{x}$ component of the ion bulk flow $(\mathrm{km} / \mathrm{s})$. Color-codes are assigned according to logarithms of counts per sample (every 4 spins) in the $E$ - $t$ diagrams.

sheet boundary.

The magnetic field was directed northward all through the plot, and the $B_{z}$ component is cnhanced in the boundary layer (Fig. 1). When densities in the boundary layer are plotted versus $B_{z}$, both are seen to be correlated (not shown). As the higher density is likcly to indicate the satcllite's closer approach/exit to the magnctosheath, this suggests that the $B_{z}$ component is positive in the magnetosheath, and is larger than in the adjacent boundary layer (IMF $B_{z}$ obtained by WIND at $X_{G S M}=5 R_{E}$ was positive $(+5 \mathrm{nT})$ around $\left.15 \mathrm{UT}\right)$.

Until 14:28 UT, Geotail was in the plasma sheet. This is known from the presence of hot ions and electrons with low density $(0.1 / \mathrm{cc})$. At 14:28 UT, changes in the three E-t diagrams (Fig. 2) are recognized. After this time, as is readily seen in the $E$ - $t$ diagrams for the tailward ions, blobs of cold-dense plasma flowing tailward were frequently encountered, with the plasma sheet appearing when the cold plasma are not present. The density of cold plasma inside these blobs were $>0.5 / \mathrm{cc}$, higher than the plasma shect value and is highest around 15:30 UT (possibly in the magnetosheath). The $E$ - $t$ diagram of electrons shows that electrons of $>100 \mathrm{eV}$ are depleted when the ion tailward flow is siginificantly large. As the energetic electrons are of the magnetospheric origin, their depletion suggests that fast tailward ions are on open field lines.

Figure 3a shows the density-temperature $(n-T)$ correlation of the ions detected in the boundary layer. Figure $3 \mathrm{~b}$ shows the same for the plasma sheet dataset (data points obtained during 

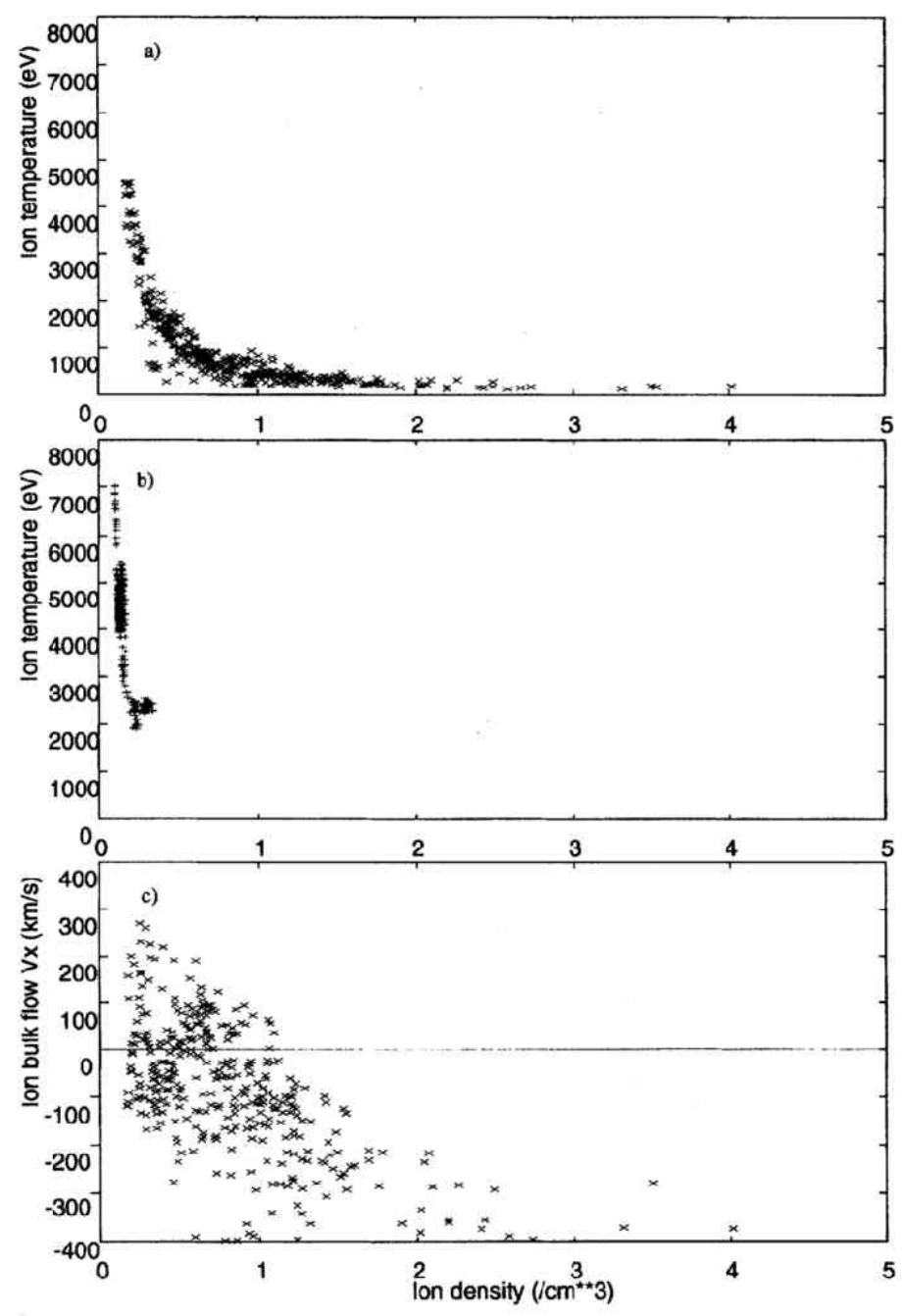

Fig. 3. (a) A plot showing correlation between the ion density (/cc) and the ion temperature (eV) in the boundary layer. Two are anti-correlated. (b) The same as Panel (a), but for plasma sheet data. (c) Correlation between the ion density (/cc) and the ion bulk flow component $V_{x}$ in the boundary layer. Data points with density $>0.5 /$ cc having positive $V_{x}$ are identified.

13:30-14:15 UT and 16:15-16:30 UT intervals). In Fig. 3a, we can clearly see that the two parameters are anti-correlated with each other, which is unexpected from a usual thermodynamical evolution conserving the entropy. When we compare Fig. 3a with Fig. 3b, it is evident that the hot-tenuous end of the data points in the boundary layer occupy mostly the same area as the plasma sheet on the $n-T$ plane. On the other hand, $(n, T)$ of the cold-dense end resembles that of the magnetosheath plasma. This suggests that the boundary layer ions have been transported from two different sources, magnetospheric convection providing the hot and tenuous plasma, and plasma entry from the adjacent flank of the magnetotail providing the other cold and dense plasma. The data points between the two ends are distributed so as to satisfy the relation $n T=$ const. As the magnetic field intensity did not change much during the boundary layer interval, this relation is needed to be satisfied to kecp the boundary layer in pressure balance. It is 
likely that the boundary layer plasma is the mixture of the plasma sheet and the magnetosheath plasma, and the mixing process is subject to the constraint of constant plasma pressure.

While a brief inspection of the ion $E$ - $t$ diagrams in Fig. 2 shows that these cold ions are more often found, and is enhanced, in the panel for the tailward flowing component, a closer look at the panels reveals that they can also be found in the sunward flowing components. Furthermore, in some of these cases (marked by vertical bars in Fig. 2), the total ion bulk velocity is directed sunward. Figure 3c, showing the $n-V_{x}$ correlation of the ions in the boundary layer, demonstrates this clearly. There is a trend that $V_{x}$ increases tailward as the density increases, consistent with the idea that higher density reflects the satellite's closer approach/exit to the magnetosheath. (The solar wind data obtained by WIND showed $n \sim 4 / \mathrm{cc}$ and $V_{x} \sim-600 \mathrm{~km} / \mathrm{s}$.) The data points are, however, so scattered about this trend that some of the data having intermediate density $(0.5-1.0 / \mathrm{cc})$ are seen to have positive $V_{x}$. Figure 3a indicates that the temperature for these data points are below $1 \mathrm{keV}$, and there should be considerable magnetosheath plasma contained in these sunward convecting flux tubes.

A reasonable interpretation of Fig. $3 \mathrm{a}$ is that the density (or the temperature) is an indicator of the degree of plasma mixing. Here, let us consider that the mixing process would produce a stratified layer and the degree of the mixing at a point in space is indicative its relative location in the boundary layer. Pulsive appearances in time series (see Fig. 1) are attributed to the fast motion of the boundary, which is manifested in the $V_{y}$ panel of Fig. 1. Using the density as a pseudo-spatial parameter in this way, the correlation in $n-B_{z}$ (not shown), $n-T$, and $n-V_{x}$ leads to an understanding of the boundary layer structure as follows. Here, as it was not possible, no discrimination between connected open field lines and interplanetary field lines (magnetosheath field lines) is made. The region termed as the outer part of the boundary layer may include data taken in the magnetosheath, but this will not affect the conclusion of the present paper significantly. The outer part (with higher density) is characterized by low temperature plasma flowing tailward. The electron data suggests that the field lines are open. The $B_{z}$ component is higher compared to the inner part. The temperature becomes higher in the inner part and the average flow velocity becomes slower. At the point where the density is less than 1 , some data come to have positive $V_{x}$, despite the fact that the value of the density indicates that there is substantial contribution from the magnetosheath population in this part of the boundary layer.

Electron anisotropy gives us further information on the structure of the boudary layer. Figure 4 shows the pitch angle anisotropy of electrons for the same interval. Differential fluxes of selected energy channels are sorted by pitch angles, and are color-coded and plotted versus time. The most obvious feature in this figure is the bi-directional anisotropy of electrons $200 \mathrm{eV} \sim 500$ $\mathrm{eV}$ in the boundary layer. (Actually, the end time of the boundary layer interval, 15:42 UT, is determined from the time the bi-directionality ceases to appear.) Among bi-directional thermal electrons that have been detected at various parts of the magnetosphere, here let us refer only to those that have close relevance to the present study: They are bi-directional thermal electrons in the inner part of the dayside LLBL (Ogilvie et al., 1984; Hall et al., 1991), and at the tail flank (Traver et al., 1991). Comparing the ion bulk velocity $V_{x}$ and the pitch angle anisotropy, we found that enhanced flux in the parallel/anti-parallel direction for the $200 \mathrm{eV} \sim 500 \mathrm{eV}$ energy range is only detected when $V_{x}$ is not strongly tailward. On the other hand, as mentioned previously, when $V_{x}$ is strongly tailward, electrons of $>100 \mathrm{eV}$ are seen to be depleted, suggesting open topology of the field lines.

The distribution function of the bi-directional electrons (Fig. 5a) suggests that the anisotropy character is produced as the cold magnetosheath electrons are heated in the parallel direction. The correlation between the bi-directional character and the slow tailward flow suggests that the electron parallel heating takes place as ions that used to flow tailward are slowed down. It should be further noted that the flux in both directions are well balanced. As in Traver et al. (1991), we will take this fact to be suggesting the closed topology of the field lines. This idea, based solely 


\section{GEOTAIL - LEP Electron Pitch Angle Distribution} Differential - Flux Plot 941225
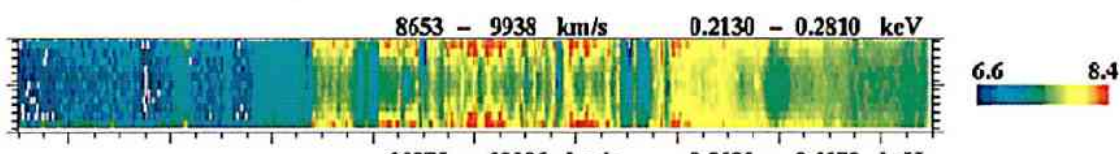

$11075-12106 \mathrm{~km} / \mathrm{s}$

$0.3491-0.4172 \mathrm{keV}$
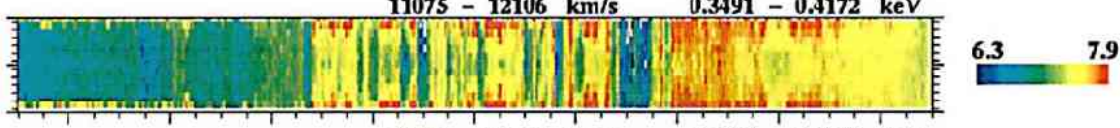

$13056-14492 \mathrm{~km} / \mathrm{s} \quad 0.4853-0.5981 \mathrm{keV}$
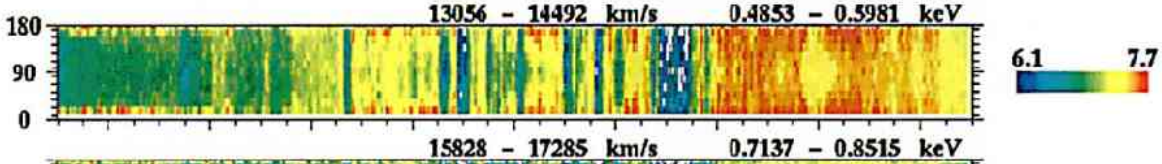

5.9

7.9
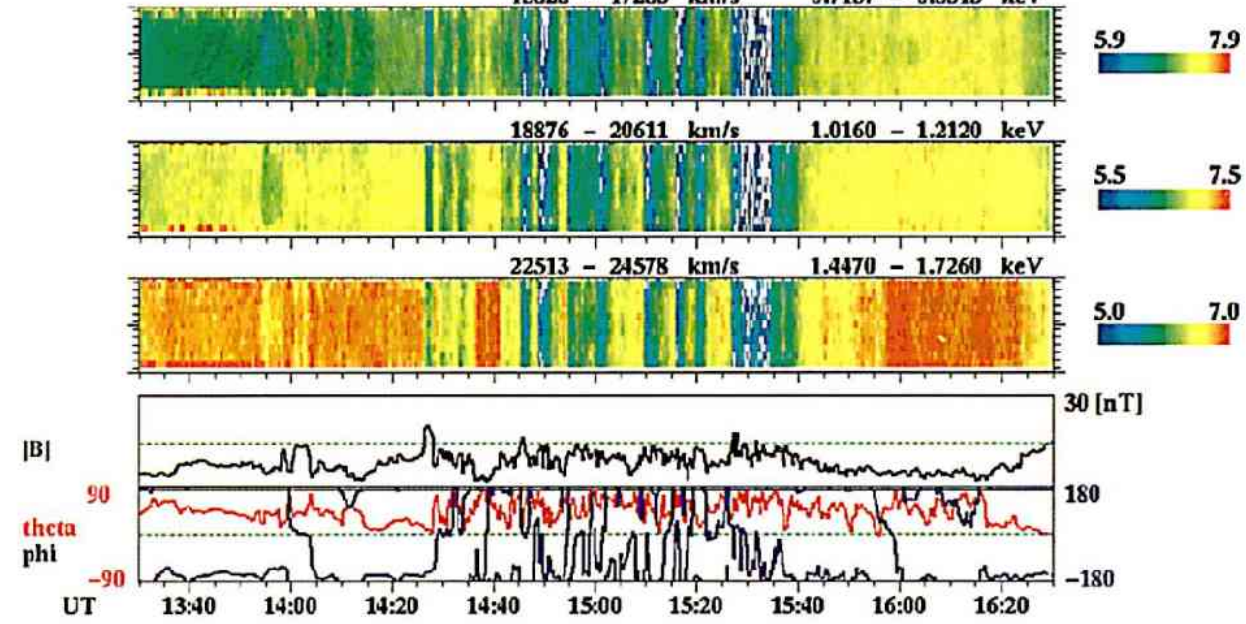

Fig. 4. Electron pitch angle anisotrpy for 13:30-16:30 UT. Differential fluxes $\left(\mathrm{cm}^{2} \cdot \mathrm{str} \cdot \mathrm{sec} \cdot \mathrm{keV}\right)$ of slected energy channels are sorted by pitch angles and plotted versus time. Color codes are assigned according to logarithms of fluxes. Bi-dircetional anisotropy at $200500 \mathrm{eV}$ in the boundary layer, and bi-directional anisotropy at $>500$ $\mathrm{eV}$ in the adjacent plasma shect (13:30-13:45 UT) are clearly seen.

on the anisotropy of electrons, should not be taken as the only possibility. The most obvious counter-example is the polar rain in the lobes of the distant tail (Baker et al., 1986), in which bi-streaming electrons are formed on open ficld lines through the mirroring effect at low altitude. See also Fuselier et al. (1995). This point will be revisited later.

Another feature we notice in Fig. 4 is the bi-directional anisotropy of electrons $>500 \mathrm{eV}$ in the plasma sheet ( 13:40 UT). In contrast to those found in the boundary layer, this distribution in the plasma sheet is characterized by its highly-collimated character. Electrons of such character has been observed at the radial distance of $10 R_{E}$ by Klumpar et al. (1988). In Fig. 5, we compare the electron distributions in the boundary layer and the plasma sheet. The two samples are taken from the intervals where the two types of bi-directional anisotropics discussed above were seen to highly develop, respectively. Shown on the left are the 3D electron distribution sliced by a plane including the magnetic field and the ion bulk flow vectors. The $B$ axis points to the magnetic field direction, and the $C$ axis to the perpendicular velocity direction. Cuts of this slice along the $B$ (red curve, parallel spectrum) and $C$ axes (bluc curve, perpendicular spectrum) are shown on the right. 
a)

cut at 15:05:18

De BCE slice (distr.fn.): $\max =-14.5 \mathrm{~min}=-19.5$
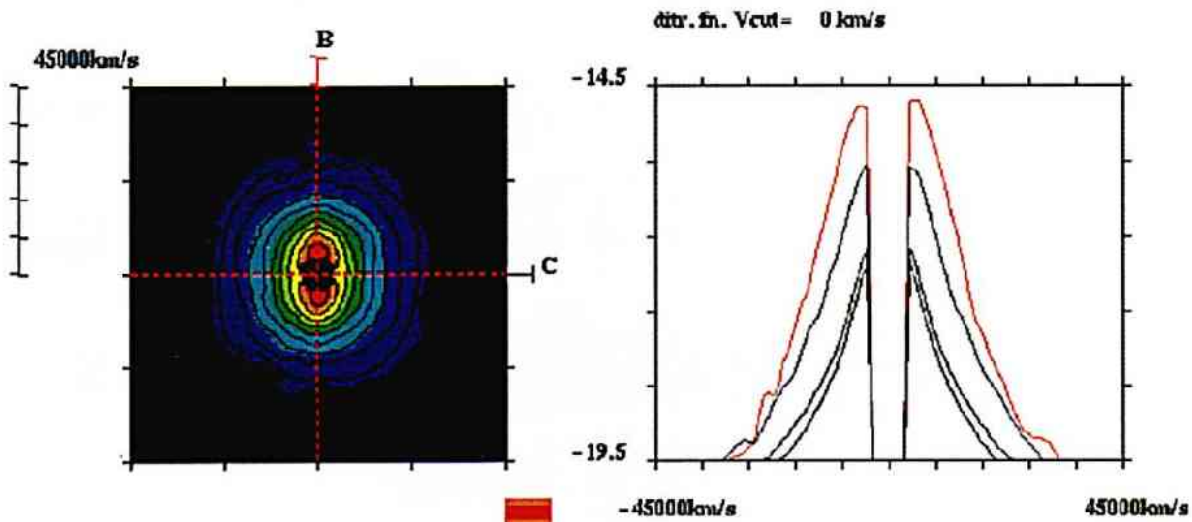

$B=(-0.284,-0.248,0.926)$

C- $(0.923,-0.334,0.194)$

b)

cut at 13:35:45

Ele BCE slice (distr. fn.): $\max =-16.5 \mathrm{~min}=-20.5$
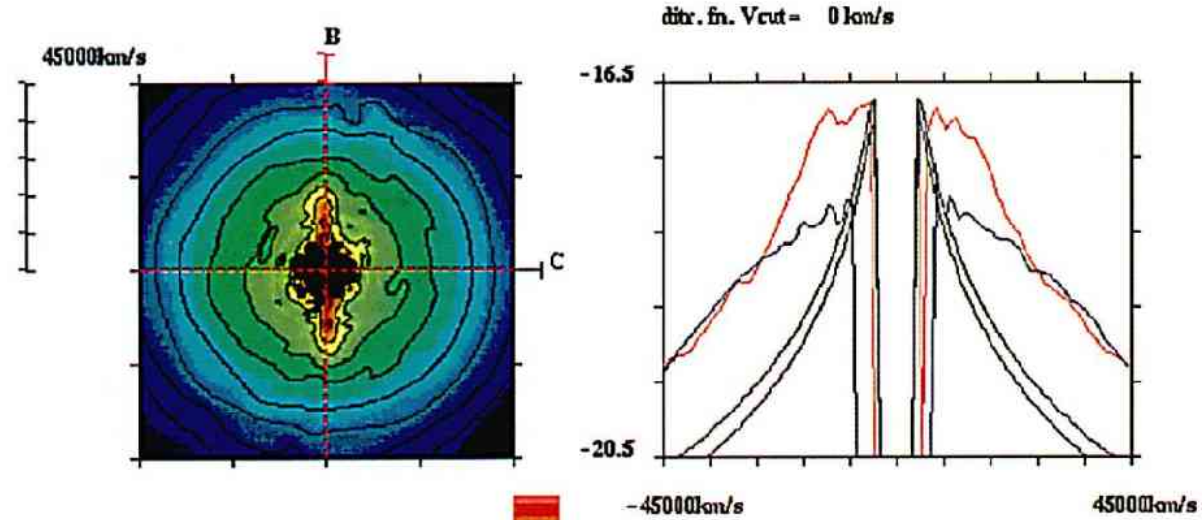

$B=(-0.255,-0.253,0.933)$

$C=(0.958,-0.193,0.210)$

Fig. 5. Electron distributions (a) in the LLBL and (b) in the plasma sheet. Slices of 3D distribution data by a plane including the magnetic field and the ion bulk velocity vector are shown on the left. The $B$ axis points to the magnetic fied direction, and the $C$ axis to the perpenicular flow direction. Shown on the right are cuts of these distributions along the $B$ axis (red) and the $C$ axis (blue). 
The sample from the boundary layer (Panel a) shows that the parallel component is enhanced for electrons of $<1 \mathrm{keV}$, and is isotropic above this energy. As mentioned before, electrons flowing along and against the magnetic field are well balanced. The spectrum suggests a parallel heating of cold isotropic magnetosheath electrons to be producing the electrons $<1 \mathrm{keV}$. The magnetospheric component is populating those in the higher energy range, though the flux level is considerably reduced from the original magnetospheric level (see Panel b).

Striking contrasts with this, not only in the picth angle character but also in the parallel energy spectrum, are shown by the plasma sheet electrons (Panel b). The parallel spectrum (red curve on the right) having bumps below $\sim 1 \mathrm{keV}$ is not expected to result from a slow heating process. Instead, as an acceleration process is applied only to those electrons having relatively small pitch angles, that is, having their mirror points at relatively low altitude, it is likely that these electrons have experienced the acceleration process inside the auroral region. Klumpar et al. (1988), studying similar electrons, suggested that the electrons are a component released from beneath the auroral parallel potential drop under dynamic auroral conditions. The electrons can also be produced if they are strongly accelerated in the perpendicular direction at their mirror points in the auroral region. The gained perpendicular energies will be turned into the parallel at the equator by magnetic focusing. In contrast to the adjacent plasma sheet, no positive proof for the mapping to active auroral regions was found in the boundary layer electrons. This may be worth keeping in mind when one is to evaluate a theoretical model dealing with the magnetosphere-ionosphere coupling.

Detection of magnetosheath-like ions convecting slowly tailward, or even sunward sometimes, in the inner part of the tail-LLBL suggests that it is actually the site where some of the plasma flowing past is captured onto the closed field lines of the magnetosphere. If only the slowing down is observed, it may be accomplished even on open field lines by the ionospheric drag acting at one end of the field line. To explain the sunward flow, however, not just the resistive load but field line tension directed sunward is necessary. Kelvin-Helmholtz instability (e.g., Miura, 1987), driven unstable at the magnetopause, may produce sunward flowing open flux tubes, but the observed change in the electron characteristics both in energy spectrum and in pitch angle will not be reproduced. Assumption of the closed topology not only fulfills the requirement for sunward tension, but is also consistent with the detection of bi-directional electrons there, and is also consistent with the significant transition in the electron spectrum. Magnetospheric electrons, though reduced from its original level, are seen trapped in the slowly convecting flux tubes, which is possible on the closed field lines. Some modeling consideration on the formation mechanisms of the boundary layer will be given later.

Our observation, fast tailward flowing open flux tubes in the outer part and slow tailward/sunward convection of closed field lines in the inner part, is in reasonable agreement with the previous studies at $X_{G S M}>-15 R_{E}$ (Williams et al., 1985; Mitchell et al., 1987). It is also noted that this example is representative of a group of data from the tail dawnside LLBL at $-30 R_{E}<X_{G S M}<-15 R_{E}$ obtained to date by GEOTAIL. Although the small dataset does not allow a statistical study of, say, the IMF $B_{z}$ dependence, we may conclude that formation of the boundary layer on closed field lines, or the plasma entry onto the closed field line from the flanks, seems, at least, not a rare event.

\section{Plasma Sheet Observation}

Having an evidence that plasma entry onto the closed field lines is taking place at the flanks of the tail (leaving aside the discussion on how), we can proceed to investigate how much this population contributes to the plasma budget in the magnetotail. As a first step, we study a case that suggests that the plasma from the flanks can sometimes fill a substantial part of the plasma sheet well inside the magnetopause. 


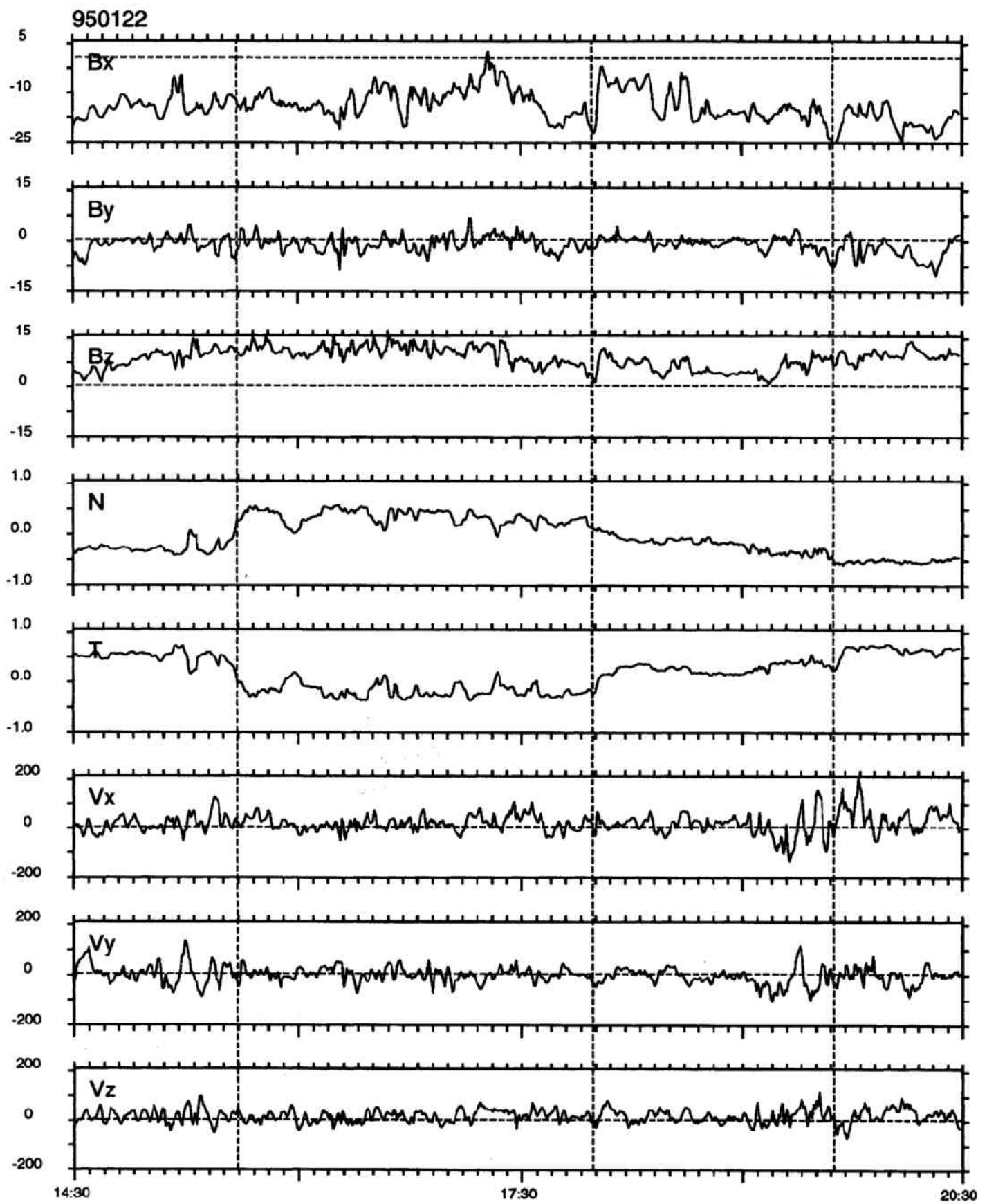

Fig. 6. Overview of the data in the plasma sheet at $\left(X_{G S M}, Y_{G S M}\right)=(-17,-12) R_{E}$. Ion density and temperature are plotted in logarithmic scale. Three major step-wise jumps in ion density and ion temperature are marked by dashed lines. 


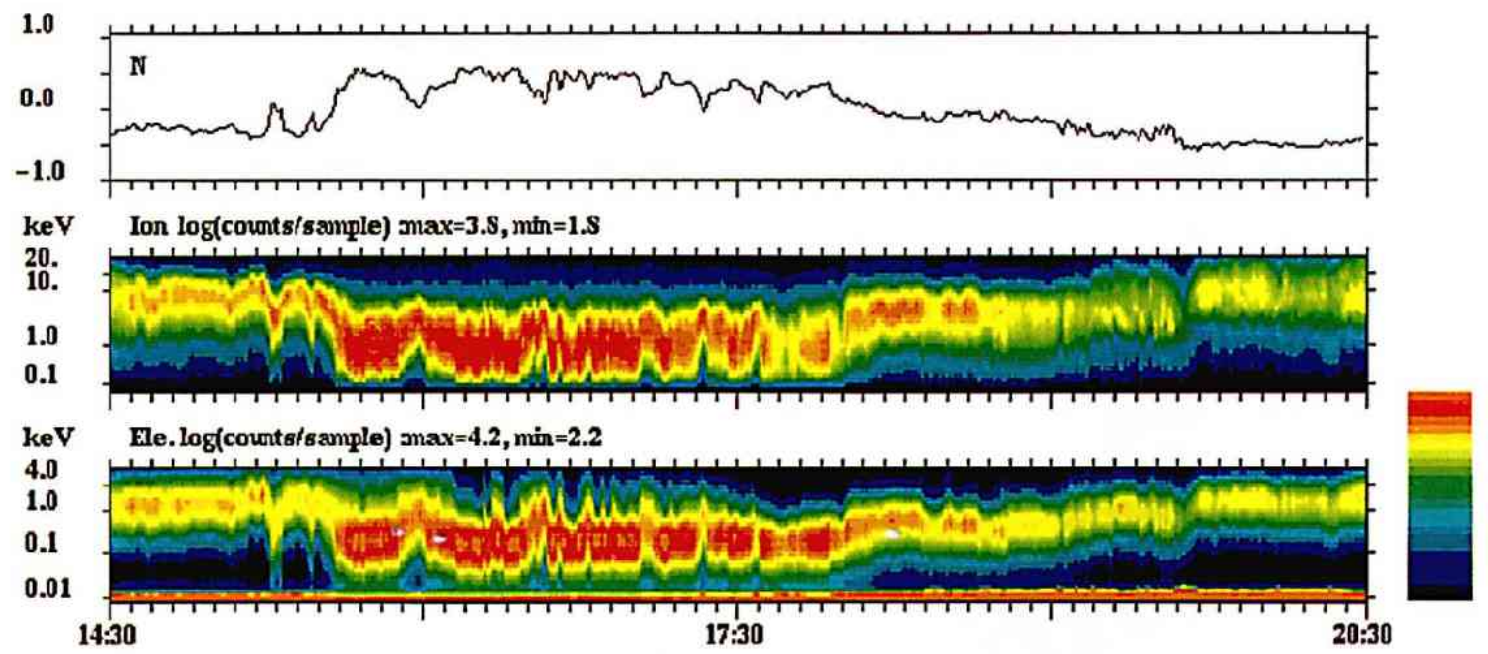

Fig. 7. E-t diagrams for omnidirectional ions and electrons. Lower energy particles appear when the density is elevated.

Figure 6 shows the 6-hours interval that will be studied. Geotail was located around $\left(X_{G S M}, Y_{G S M}, Z_{G S M}\right)=(-17,-12,-4) R_{E}$. Shown are the three components of the magnetic field, the threc components of the ion bulk flow, the ion density and the ion temporaturc. Figure 7 shows $E$ - $t$ diagrams for omnidirectional ions and electrons, compared with ion density. In this interval, three major step-like variations in the ion density and the ion temperature can be seen. These are marked at 15:35, 18:00, and 19:36 UT (vertical dashed lincs in Fig. 6). The first change is from a hot-tenuous $(T, n)=(5 \mathrm{keV}, 0.3 / \mathrm{cc})$ to a cold-dense $(0.5 \mathrm{kcV}, 3 / \mathrm{cc})$ situation. The next change is from the prior to one that has an intermediate character, and the final change causes GEOTAIL to re-encounter the hot-tenuous plasma that was observed at the beginning of the interval. As we can see in the $E$ - $t$ diagram (Fig. 7 ), the clectron energy also changed accordingly.

Figure 8 shows the density-temperature $(n-T)$ corrclation of the ions for the interval. As is clear, the parameters are anti-correlated with each other. The data points are distributed more or less along a curve between the points $(n, T)=(3 / \mathrm{cc}, 500 \mathrm{eV})$ and $(0.3 / \mathrm{cc}, 5000 \mathrm{eV})$. As in the boundary layer case, the curve goes so as to satisfy the relation $n T=$ const. While the hot-tenuous end is about the nominal plasma sheet value expected at this location (c.g., Baumjohannet al., 1989), the cold-dense end is similar to what is detected in the inner part of the boundary layer at the flank, that is, the part of the LLBL on closed field lines (see the former section). It should be mentioned, however, that the GEOTAIL position for the above interval was well inside the nominal magnctopause so that the encounter with the cold-dense ions cannot be due to crossing into the boundary layer. The facts that three components of the magnetic field are rather steady, and that the ion bulk flow is steadily slow, suggest that the appcarance of boundary layer-like plasma is not due to GEOTAIL's crossing into any different region (LLBL, mantle, and so on). A natural interpretation is that the encounter with the cold-dense ions took place within the plasma sheet.

Figure 9 shows the electron pitch angle anisotropy for the above interval. The format of the figure is the same as Fig. 4. What is most remarkable in this figure is the appearance of the bi-directional character of thermal component in the 2.5 hours interval where the cold-dense ions were detected (15:35-18:00 UT). Not only the ion moments, but also the electron anisotropy in this cold-dense part are similar to the data obtained in the boundary layer. Eastman et al. (1985) 


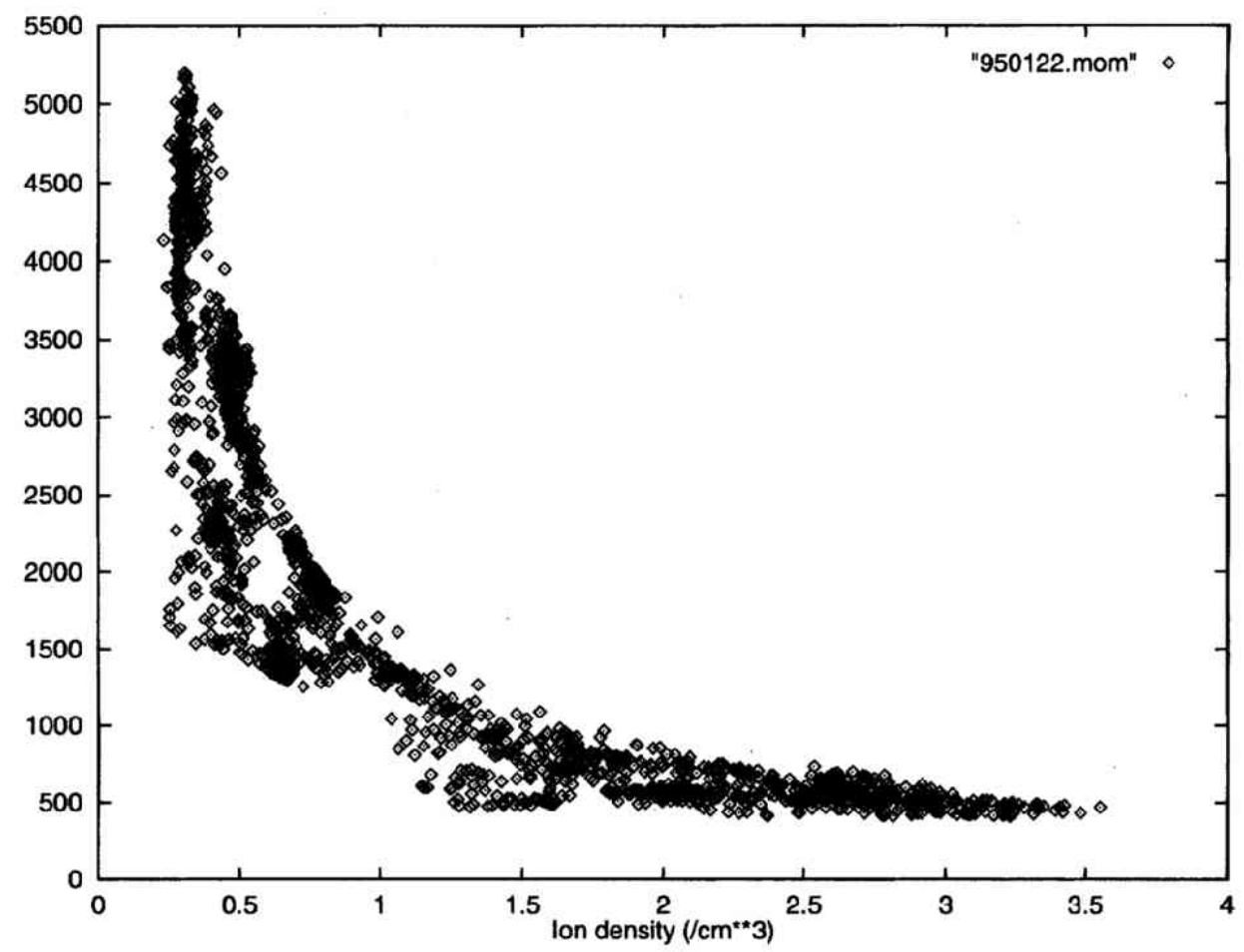

Fig. 8. Density-temperature correlation for the interval 14:30-20:30 UT. Anti-correlation of the two is clear.

already interpreted the cold ions occasionally detected in the central plasma sheet to be supplied from the flank boundary layers. Here, our data showing bi-directional electrons co-existing with the cold-dense ions, strengthens the argument: From the close similarity of the data, we also interpret that the plasma in the cool-dense part is what has entered the magnetotail from the flank on the dawnside, and been convected to the observed location. The low ion temperature is comparable to that in the boundary layer, suggesting that they have remained unheated on their way being transported from the entry location. Assuming that the adiabatic theory gives a reasonable estimate to the amount of heating, the intensity of the magnetic field at the entry point and in the plasma sheet $(15 \mathrm{nT})$ should be comparable. This suggests that the entry location should not have been too far down the tail. It may be worth noting that the magnetic field in the boundary layer at $X_{G S M}=-27 R_{E}$ was actually $15 \mathrm{nT}$ (see the former section). In contrast, hot-teneous plasma are likely to be from the distant tail source, which have experienced significant heating while being transported.

The fact that the density and temperature of the cold-dense part is unchanged from the boundary layer plasma suggests that this core part is filled exclusively with the unheated plasma from the boundary. It is likely that the plasma in the core part is convected as a mass, rather than diffusively transported (Eastman et al., 1985), nor has magnetically drifted (Spence and Kivelson, 1994), from the entry point. The accompanying bi-directional electrons is consistent with this idea. As the core part is expected to have a convection character different from the surrounding regions (to be specific, enhanced dawn-to-dusk motion), we have inspected the data, but curiously, found nothing. On the other hand, Fig. 8 suggests that some mixing processes proceed at the interface between the cold-dense core and the surrounding hot-teneous plasma (under the constraint of constant plasma pressure). The density and temperature variations in 


\section{GEOTAIL - LEP Electron Pitch Angle Distribution} Differential - Flux Plot 950122

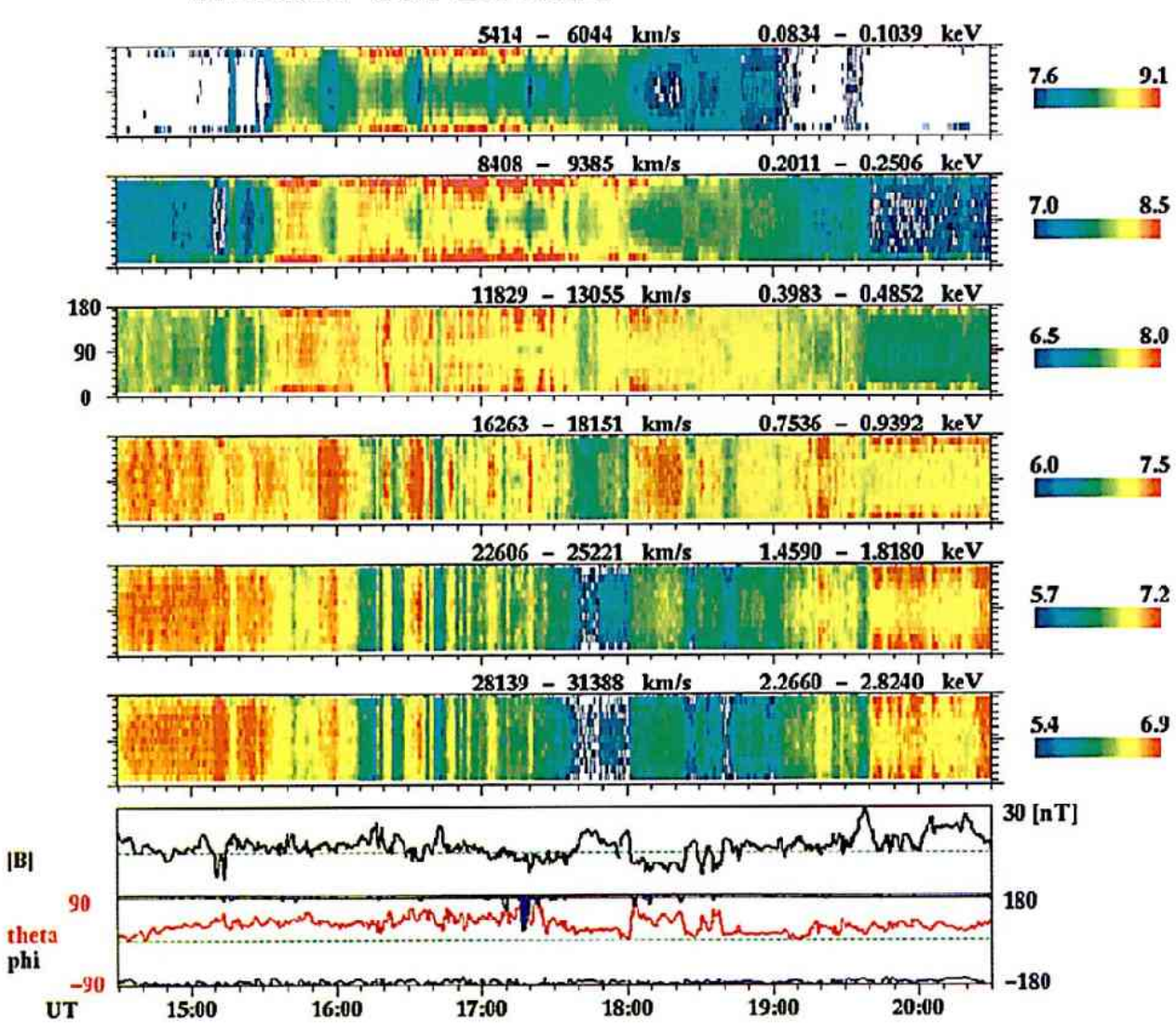

Fig. 9. Flectron pitch angle anisotropy for 14:30-20:30 UT. Bi-directional anisotropy similar to those in LLBL is detected in the cold-dense part of the plasma sheet. Bi-directional anisoptropy of more energetic component is detected after 19:40 UT.

Fig. 6 are characterized by step-wise jumps from one plasma to another, and as they are not necessarily associated with fast plasma flow, they indicate that the width of the mixing layer is limited and the two plasmas do not easily mix with each other. On the other hand, the prolonged period of a region of intermediate characters (18:00-19:36 UT) suggests a formation of a spatially extended mixing region. Understanding the nature of the mixing process definitcly needs further study.

Another correspondence with the boundary layer data seen in the electron pitch angle anisutropy (Fig. 9) is the bi-directional electrons of totally different characters, which is suggestive of an auroral acceleration, detected in the hot-tenuous part. This is most evident in the panels for electrons of $>750 \mathrm{eV}$ after 19:40 UT. Figure 10 compares both the bi-directional electrons. The striking difference is just what was seen in the tail LLBL. The parallel spectrum in Panel a, obtained in the cold-dense part, has a smooth shape and exceeds the perpendicular spectrum at energies below $\sim 500 \mathrm{eV}$. The parallel spectrum from the hot-teneous part in Panel b has two clear bumps at $\pm 9,000 \mathrm{~km} / \mathrm{s}$, suggesting that they are related to active aurora. 
a)

cut at 17:03:34

Ele BCE slice (distr. fn.): $m a x=-14.5 \mathrm{~min}=-19.5$

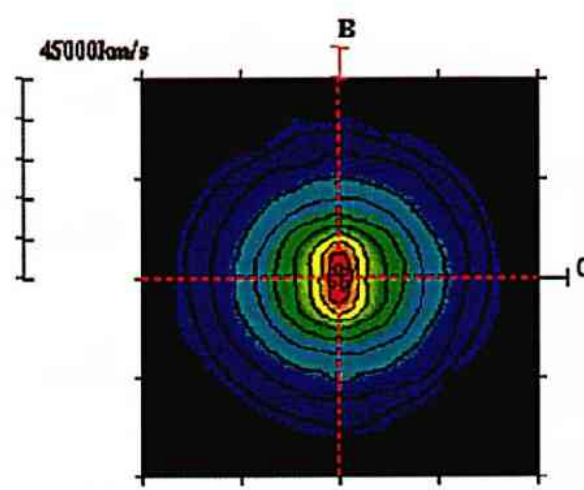

ditr.fn. Vcut- $0 \mathrm{lom} / \mathrm{s}$

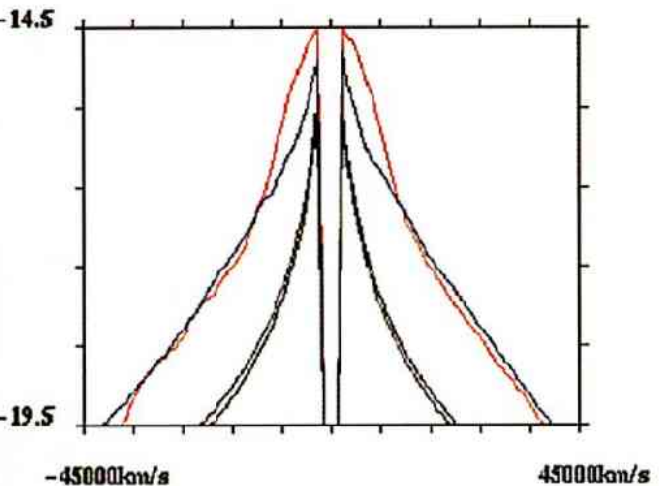

$B=(-0.569,-0.112,0.815)$

$C=(-0.131,-0.966,-0.224)$

b) cut at 20:06:05

Ele BCE slice (distr, fn.): $\max =-16.0 \mathrm{~min}=-21.0$
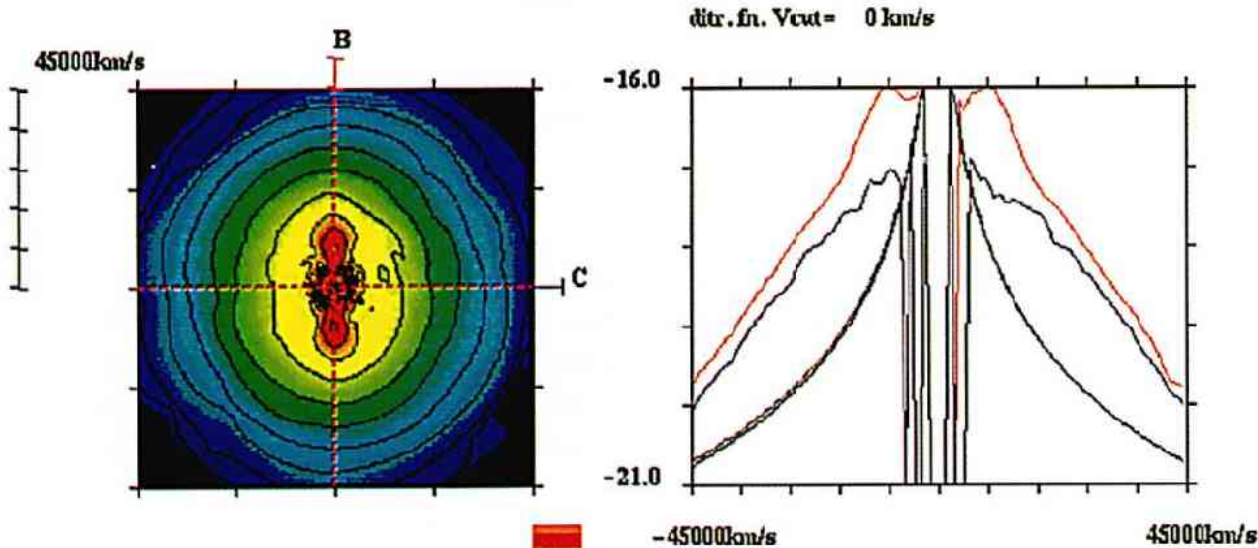

$B=(-0.907,-0.174,0.393)$

$\mathrm{C}=(0.398,-0.648,0.650)$

Fig. 10. Comparison of the two different bi-directional electrons detected in the plasma sheet. (a) In the cold-dense part, and (b) in the hot-tenuous part. 


\section{Summary and Discussion}

The observation of the LLBL on dawnside at $X_{G S M}=-27 R_{E}$ can be summarized as follows: It is where magnetosheath-like cool-dense plasma appears. The outer part is characterized fast tailward flow. The energetic electrons are seen to be depleted in this part, suggesting an open topology of the field lines. (Some data points in the outer part may actually be obtained in the magnetosheath. Discrimination between the two was not made in this paper.) In the inner part of it, these cool ions have only slow tailward convection velocity, and is even flowing sunward sometimes. In this slowly convecting part, electrons of $200 \sim 500 \mathrm{eV}$ are enhanced in the fieldaligned direction, with fluxes in both the parallel/anti-parallel direction being balanced. Slow convection and this heated bi-directional electrons suggest the closed topology of the field lines in this part of the boundary layer. It turned out that our example shown here summarizes a group of data from the tail dawnside LLBL obtained by GEOTAIL to date. In addition to these characteristics, duskside cases showed more clear evidences of ion mixing: The two components (magnetospheric and magnetosheath) are well separated in energy and as the both show peaks in count rates, a double-band structure is visible in the $E$ - $t$ diagram of ions. Such was not seen in the dawnside cases, and the origin of this dawn-dusk asymmetry seems to be the cross tail magnetic drift of the plasma sheet ions, bringing more energetic ions out to the duskside boundary layer. Further results will be reported elsewhere.

In the inner-LLBL, the cold-dense ions originated from the magnetosheath are captured on closed field lines. One of possible formation mechanisms of this boundary layer is the reconnection of open field lines, which have one of their ends in the ionosphere, and, on which the magnetosheath plasma are freely streaming. A recent study of the dayside LLBL (Fujimoto et al., 1996) has shown that such open field lines are formed as the reconnected field lines are draped azimuthally around the magnetospheric obstacle, and constitute the outer part of the dayside LLBL away from the subsolar region. This outer-LLBL is likely to form as long as there is finite IMF $B_{y}$ component, and unless IMF $B_{z}$ is strongly southward so that peeling of the reconnected flux tubes in north-south direction is overwhelming. In the magnetotail LLBL, tailward ions, that used to be freely streaming along the open, but now find themselves on closed field lines, are slowed down and eventually turned sunward due to the field line tension. It should be noted that, since the Alfven velocity in the sheath/boundary layer region was only $\sim 150 \mathrm{~km} / \mathrm{s}$ for the case studied, the acceleration associated with the reconnection alone is too small to turn the magnetosheath ions sunward, and further sunward tension acting on the newly reconnected closed field lines is necessary. The reconnection might also heat electrons in the parallel direction (Fuselier et al., 1995). According to this model, slowly tailward convecting closed field lines at $X_{G S M}=-27 R_{E}$ would mean that the reconnection at the flank is already taking place at locations further earthward.

The above model explains that the inner boundary layer is formed as closed flux tubes are added at the flanks of the magnetotail. Alternatively, the boundary layer may be formed as magnetosheath plasma is added to the closed flux tubes of the magnetotail. The ion mixing driven by K-H instability (e.g., Fujimoto and Terasawa, 1994) is one of the possibility. Once the magnetosheath ions are on closed field lines, their slowing down and eventual sunward turning are described in the MHD models of viscous LLBL (e.g., Phan et al.,1989; Drakou et al., 1994). The $V_{x}$ component in the inner boundary layer are scattered around the average value. This could be indicating formations of vortices that would activate the viscous-like interaction.

Bi-directional thermal electrons are also detected in the inner part of the dayside LLBL (e.g., Hall et al., 1991; Fujimoto et al., 1996). It may be considered that the electrons of the same anisotropy in the tail may have been convected from the dayside. As opposed to a simple minded idea that the flow in the LLBL should be dragged tailward, however, it is recently found that the convection in some of the dayside inner-LLBL tends to be sunward (Fujimoto et al., 1996). 
This even suggests the opposite idea that plasma in the dayside inner-LLBL are (sometimes) transported from its tailward extension. Not only the questions, at what $X_{G S M}$ the plasma entry takes place in the tail boundary layer, and, what is the entry mechanism, but also its connection to the dayside part must be clarifed in a future study.

How much does the plasma thus captured into the magnetospheric closed field lines populate the plasma sheet? Results presented in the former section implies that it cannot be neglected at least. In the plasma sheet at $\left(X_{G S M}, Y_{G S M}\right)=(-17,-12) R_{E}$, cold-dense ions were detected for about 2.5 hours. Not only the ion temperature and density, but also the thermal electron pitch angle anisotropy were similar to those found in the tail LLBL. From these similarities, it is interpreted that this plasma has come from the dawnside flank of the tail. On the basis of the adiabatic theory, and from the unheated feature of the cold-dense ions, it is inferred that their entry points are not too far down the tail. The core part of the cold-dense ions were not mixed with the usual hot plasma sheet component, and should be contrasted with the inner-LLBL at the dayside (e.g., Sckopke et al., 1981). What this means is that the plasma from the flank is convected as a mass and almost exclusively fill a substantial part of the plasma sheet sometimes. The plasma mixing are seen to take place in the region surrounding the core part, but its nature remains to be investigated in the future.

This cold-dense plasma sheet was sandwiched by the nominal ones, and electrons that are likely to be related to auroral activities were detected only in the latter. Such difference was also seen between the boundary layer electrons and the adjacent plasma sheet electrons. It can be that, together with the tail-LLBL, the part of the plasma sheet occupied by the cool-dense component is mapped down to the ionosphere where only less active aurora exist. How the properties of the plasma sheet population affects the magnetosphere-ionosphere coupling process is an interesting problem that must be investigated in the future.

The ultimate goal to be reached in the study of this line, the initial results of which being reported in this paper, is to quantitatively assess the contribution of the flank entry to the plasma budget in the tail. To understand what has triggered the plasma source switch in the present case would be useful for a future study. Among various solar wind parameters that showed temporal variations around the event time, we refer to the turning of IMF to enhanced northward $B_{z}$. This choice is based on a preliminary survey (Terasawa et al., in preparation) that suggests the contribution of the flank entry to be enhance during strong northward IMF periods. This choice is also reasonable from a modeling point of view, for the distant tail source will dominate during enhanced convection intervals, and these are when the magnetosphere is under the influence of southward IMF. Further studies will be reported in the future.

M. F. acknowledges fruitful discussion with T. D. Phan. He also thanks D. N. Baker for his careful reading the manuscript. The key parameter data of WIND were provided by the NASA/GSFC data processing team. A part of this work has been suported by the Grant-in-Aid for Scientific Research (No. 07730394) from the Ministry of Education of Japan.

\section{REFERENCES}

Baker, D. N., et al., Strong electron bi-directional anisotropies in the distant tail: ISEE 3 observations of polar rain, J. Geophys. Res., 91, 5637-5662, 1986.

Baumjohann, W., G. Paschmann, and C. A. Cattell, Average plasma properties in the central plasma sheet, $J$. Geophys. Res., 94, 6597-6606, 1989.

Drakou, E, B. U. O. Sonnerup, and W. Lotko, Self-consistent steady state model of the low-latitude boundary layer, J. Geophys. Res., 99, 2351-2364, 1994.

Dungey, J. W., Interplanetary magnetic field and the auroral zone, Phys. Rev. Lett., 6, 47-48, 1961.

Eastman, T. E., L. A. Frank, and C. Y. Huang, The boundary layers as the primary transport region regions of the Earth's magnetotail, J. Geophys. Res., 90, 9541-9560, 1985.

Fujimoto, M., and T. Terasawa, Anomalous ion mixing within an MHD scale K-H vortex, J. Geophys. Res., 99, 
$8601-8613,1994$.

Fujimoto, M., et al., Structure of the low-latitude boundary layer: A Geotail observation, submitted to J. Geophys. Res., 1996.

Fuselier, S. A., B. J. Anderson, and T. G. Onsager, Particle signatures of magnetic topology at the magnetopause: AMPTE/CCE observations, J. Geophys. Res., 100, 11,805-11,821, 1995.

Hall, D. S., et al., Electrons in the boundary layer near the dayside magnetopause, J. Geophys. Res., 96, 7869-7891, 1991.

Kokubun, S., et al., The GEOTAIL magnetic field experiment, J. Geomag. Geoelectr., 46, 7-21, 1994.

Klumpar, D. M., J. M. Quinn, and E. G. Shelley, Counter-streaming electrons at the geomagnetic equator near 9 $R_{E}$, Geophys. Res. Lett., 11, 1295-1265, 1988.

Mitchell, D. G., et al., An extended study of the low-latitude boundary layer on the dawn and dusk flanks of the magnetosphere, J. Geophys. Res., 92, 7394-7404, 1987.

Miura, A., Simulation of KH instability at the magnetospheric boundary, J. Geophys. Res., 92, 3195-3206, 1987.

Mukai, T., et al., The low energy particle experiment onboard the GEOTAIL satellite, J. Geomag. Geoelectr., 46, 669-692, 1994.

Ogilvie, K. W., R. J. Fitzenreiter, and J. D. Scudder, Observations of electron beams in the low-latitude boundary layer, J. Geophys. Res., 89, 10,723-10,732, 1984.

Phan, T. D., B. U. O. Sonnerup, and W. Lotko, Self-consistent model of the low-latitude boundary layer, $J$. Geophys. Res., 94, 1281-1293, 1989.

Sckopke, N. G., et al., Structure of the low-latitude boundary layer, J. Geophys. Res., 86, $2099,1981$.

Spence, H. E., M. G. Kivelson, Contributions of the low-latitude boundary layer to the finite width magnetotail convection, J. Geophys. Res., 98, 15,487-15,496, 1993.

Traver, D. P., et al., Two encounters with the flank low-latitude boundary layer: further evidence for closed field topology and investigation of the internal structure, J. Geophys. Res., 96, 21,025-21,035, 1991.

Tsurutani, B. T. and T. M. Thorne, Diffusion processes in the magnetopause boundary layer, Geophys. Res. Lett., 9, 1247-1250, 1982.

Williams, D. J. et al., Energetic particle observations in the low-latitude boundary layer, J. Geophys. Res., 90, $5097-5116,1985$. 